Review

\title{
Masticatory Deficiency as a Risk Factor for Cognitive Dysfunction
}

Francisco Bruno Teixeira' ${ }^{1}$, Luanna de Melo Pereira Fernandes ${ }^{1,2}$, Patrycy Assis Tavares Noronha ${ }^{3}$, Marcio Antonio Raiol dos Santos', Walace Gomes-Leal', Cristiane do Socorro Ferraz Maia², Rafael Rodrigues $\operatorname{Lima}^{1}{ }^{凶}$

1. Laboratory of Functional and Structural Biology, Institute of Biological Sciences, Federal University of Pará, 66075-900, Belém-Pará, Brazil;

2. Laboratory Pharmacology of Inflammation and Behavior, Institute of Health Sciences, Federal University of Pará, 66075-900, Belém-Pará, Brazil;

3. Laboratory of Experimental Neuroprotection and Neuroregeneration, Institute of Biological Sciences, Federal University of Pará, 66075-900, Belém-Pará, Brazil.

$\square$ Corresponding author: Rafael Rodrigues Lima, Laboratory of Functional and Structural Biology, Institute of Biological Sciences, Federal University of Pará, Street Augusto Corrêa N. 1, Campus do Guamá, Belém-Pará 66075-900, Brazil. E-mail: rafalima@ufpa.br, rafaelrodrigueslima@hotmail.com

(C) Ivyspring International Publisher. This is an open-access article distributed under the terms of the Creative Commons License (http://creativecommons.org/ licenses/by-nc-nd/3.0/). Reproduction is permitted for personal, noncommercial use, provided that the article is in whole, unmodified, and properly cited.

Received: 2013.05.30; Accepted: 2013.II.25; Published: 2014.01.10

\begin{abstract}
Several studies have demonstrated that chewing helps to maintain cognitive functions in brain regions including the hippocampus, a central nervous system (CNS) region vital for memory and learning. Epidemiological studies suggest that masticatory deficiency is associated with development of dementia, which is related to spatial memory deficits especially in older animals. The purpose of this paper is to review recent work on the effects of masticatory impairment on cognitive functions both in experimental animals and humans. We show that several mechanisms may be involved in the cognitive deficits associated with masticatory deficiency. The epidemiological data suggest a positive correlation between masticatory deficit and Alzheimer's disease. It may be concluded that chewing has important implications for the mechanisms underlying certain cognitive abilities.
\end{abstract}

Key words: Chewing, Cognition, Learning, Memory, Masticatory, Hippocampus.

\section{Introduction}

According to the United Nations, the world population will grow by $37 \%$ by mid-century, with an increase of around 9 billion individuals in absolute terms. People over 60 years old represent $32 \%$ of the world population, and for the first time in history they outnumber children. Health conditions resulting from ageing and the health services offered to the elderly, such as access to specialized dental services, should be considered together ${ }^{[1-3]}$.

The Oral Health Program of the World Health Organization encourages public health administrators to design strategies and programs for effective and affordable oral healthcare in order to provide a better quality of life for elderly people ${ }^{[4]}$.

The challenge of chronic disease management and improvement of oral and general health can only be effectively tackled through approaches focusing on risk factors. In order to address these issues in public health programs, it is important to understand the interaction between oral and general health ${ }^{[5]}$.

Several studies[6-10] have shown that some systemic factors, such as cardiovascular and respiratory diseases, nutritional changes, diabetes, postural abnormalities, osteoporosis, etc. may interfere with the stomatognathic system. However, the relationship between the central nervous system (CNS) and the stomatognathic system is still the subject of numerous investigations. In this context, the relationship between chewing - the main function of stomatognathic system - and CNS function is of considerable im- 
portance.

Tooth loss and impairments in occlusion and masticatory force are known as oral health problems without major repercussions for dentition. Nevertheless, several studies have shown an association between chewing and cognitive functions[6-10]. In this paper, we review investigations of the relationship between masticatory capacity and age-related cognitive impairment. We also discuss the implications for human health of experimental findings in this research field.

\section{Literature Review}

Kato et al.[10] first described the association between loss of molars in senile rats and spatial memory deficits. In this study, after molar extraction, animals were maintained with diet powder for 135 weeks and were subjected to behavioral testing to assess their spatial memory. Animals without molar teeth showed worse performance in behavioral tests than control animals maintained on a solid diet. This experimental study raised another important question: what caused the failure of spatial memory? Loss of teeth or absence of the masticatory act?

\section{The regulation of masticatory function on memory and learning}

Further studies using functional magnetic resonance imaging (fMRI) and positron emission tomography (PET) revealed that during mastication there is an increase in cortical blood flow ${ }^{[11]}$ and activation of somatosensory cortical areas, the supplementary motor area, insula, and other areas including the striatum, thalamus and cerebellum ${ }^{[12,13]}$. Since these regions of the CNS receive sensory information from some areas of the stomatognathic system, and control the movements and rhythm related to mastication and facial expression, they have been termed a central pattern generator ${ }^{[14]}$. The hypothesis that mastication, rather than the presence of the teeth is paramount to functional activation of those CNS areas was derived from these studies.

Other reports show that chewing immediately before learning a cognitive task increases oxygen levels in the prefrontal cortex (PFC) and hippocampus, both CNS areas important for processes of learning and memory ${ }^{[15,16]}$.

Distinct forms of memory are mediated by different CNS regions ${ }^{[17]}$. Memories can be classified as follows: (i) declarative or explicit; the ability to recall past events deliberately, hippocampus-dependent and (ii) non-declarative or procedural, also called implicit; unconsciously performed skills (motor or cognitive), mainly dependent on the striatum and cerebellum, regions in which habits are formed[18-20].
Of the areas involved in cognitive processes, the PFC has considerable importance as it receives projections from both motor and sensory areas important for learning. It has been shown that there is an intricate neuroanatomical correlation between PFC and the basal ganglia (mainly the striatum)[21].The PFC seems to be related to learning and the basal ganglia to consolidation routines in automatic habits or procedures $[22,23]$. Other reports have suggested that the basal ganglia plays a pivotal role in learning ${ }^{[24,25]}$. The hippocampus is involved in anxiety-like behaviors, as well as in memory and learning processes, as result of its connections with other limbic areas involved in emotional behaviors, including the amygdala[26,27].

The hippocampus and striatum are interconnected with areas involved in cognitive and motivational processes, such as the basal ganglia, substantia nigra, ventral tegmental area, thalamus and amygdala. It is well established that cognitive and motivational processes depend on the connections between PFC and the brain areas cited above[28-30]. The hippocampus play a key role in the functioning of these pathways $^{[19]}$.

As reported previously, chewing increases blood flow in cortical regions, including PFC, and cerebellar regions ${ }^{[11]}$. It also promotes increased cardiac activity, suggesting greater sympathetic nervous activity[31] and increases blood glucose levels and the level of arousal during a cognitive task[32,33], which is essential for the improvement of cognitive performance.

Several studies using brain imaging and memory tasks support the hypothesis that chewing increases brain function, including working and spatial memories[33-40]. Wilkinson et al.[33] reported that in humans (mean age 24.6 years old) a masticatory stimulus can improve episodic and working memories, but not attention. On the other hand, Hirano et al.[41] suggested that chewing induces an increase in arousal level and alertness as well as an effect on motor control and suggest that these effects could lead to improvement in cognitive performance.

There is also debate about the role of chewing in cognitive function. The reasons for these discrepancies are, at least in part, due to the fact that only middle-aged subjects have been used in these studies[32-34,41,42,43]. Onyper et al.[43] investigated the effects of gum chewing on cognitive function with students and reported that using chewing gum improves cognitive task performance when it is chewed for 5 minutes prior to, but not during the cognitive task; the benefits persisted for the first 15-20 minutes of the testing session. The authors suggested therefore that the benefits attributed to chewing gum were time-limited and related to mastication-induced arousal. 
The consistency of the chewing gum may contribute to the discrepancies in effects observed in studies using fMRI and PET. Onozuka et al.[12] have shown that brain regions, such as sensorimotor cortex, the supplementary motor area and the cerebellum have different blood oxygenation level-dependent (BOLD) signals when stimulated by different consistencies of chewing gum. Another study from the same research group showed increased performance on memory retrieval when elderly people (60-76 years) used chewing gum[15]. This effect was absent in younger individuals (19-26 years). Studies using similar experimental paradigms, have found increased BOLD signal intensity in the right PFC in an age-dependent manner[12,13]. On the basis of data from Ono and colleagues[9] it can be concluded that chewing is a simple non-pharmacological way of preventing senile dementia, which is often associated with cognitive dysfunctions, such as loss of spatial memory and amnesia.

Alzheimer's disease involves formation of many senile plaques of amyloid- $\beta$ and neurofibrillary tangles ${ }^{[44]}$. Ekuni et al. ${ }^{[45]}$ suggested that psychological stress induced by occlusal disharmony induces amyloid- $\beta$ expression in the rat hippocampus through glucocorticoid signaling. According to this hypothesis, reduced masticatory ability would contribute to a reduction in rhythmic and coordinated movements, leading to reduced stimulation of mechanoreceptors in the orofacial region. Decreased activity of these receptors can lead to quantitative changes in afferent inputs from sensory receptors to the CNS, resulting in alterations in signaling in the neuroanatomical pathways from the orofacial region. It follows that the reduction in masticatory activity can alter the brain circuitry in these patients ${ }^{[46]}$.

Evidences suggest that the presence of teeth and the stimulation of chewing help to retain cognitive function. This is reinforced by epidemiological studies which show that a decrease in the number of teeth decreases the use of dentures, and that a chewing lower force is directly related to dementia ${ }^{[47]}$. Yamamoto et al. ${ }^{[48]}$ reported an association between having few teeth but no dentures and lack of regular dental care, and higher risk of dementia onset in older Japanese. Nevertheless, Lexomboon et al.[49] concluded that people who use dentures did not have chewing difficulty or cognitive impairment. Other studies have suggested that decreased bite strength associated with tooth loss and reduced chewing is also a risk factor for dementia ${ }^{[6,7,50-53]}$. It is thought that there is a direct relationship between cognitive impairment in elderly women and the decrease in average number of teeth, maximum bite force, chewing capacity, and occlusal contact area[7].

\section{Masticatory hypofunction produces cognitive impairment}

Several studies have investigated the possible association between masticatory hypofunction and cognitive deficits using animal models $[10,44,45,46]$. In some experiments, authors induced loss of functionality in the molar teeth by performing extraction or reduction of the crown rather than a long-term soft $\operatorname{diet}[10,44,46]$. Animals subjected to these methods remained able to chew, but the occlusal hypofunction caused degenerative changes in their periodontal mechanoreceptors ${ }^{[54]}$ suggesting a suppression of sensory stimulation from the periodontal ligament during chewing. In these experiments, animals showed poor performance on tests of memory and learning.

Interestingly, in these studies, cognitive deficits were observed only in adult animals and preferentially in the aged animals subjected to the loss of molar functionality over a short period of about 7-10 days ${ }^{[5-60]}$. The cognitive deficit did not occur in young animals subjected to the same experimental treatment. In these studies, restoring the damaged molars with artificial crowns resulted in reversal of the learning deficits, even in older animals ${ }^{[59]}$.

Some reports provided histological data suggesting that the hippocampus is the main CNS region affected by masticatory hypofunction. The hippocampus is involved in some types of memory, especially declarative memory, which relates to facts and precise events ${ }^{[10,61]}$. With age, this brain region becomes highly susceptible to morphological[10,57] and physiological changes ${ }^{[55,62] .}$.

It has been reported that masticatory dysfunction contributes to a decrease in the density of pyramidal neurons ${ }^{[55]}$ and dendritic spines ${ }^{[60]}$, and is associated with increased astrocytosis and hypertrophy in the hippocampal CA1 field in mice ${ }^{[5,57]}$. These morphological alterations were correlated with spatial memory impairment in a water maze test. The observation of hypertrophic astrocytes suggests inflammation and degeneration. In these studies, activated microglia seems to release pro-inflammatory cytokines, which may contribute to hippocampal damage ${ }^{[63,64]}$. Astrocytes may also be damaged, contributing to impairments in neurotransmitter uptake and release of growth factors which have important effects on neurotransmission and the mechanisms underlying learning and memory ${ }^{[65]}$.

Masticatory hypofunction may also affect neuroplasticity. Watanabe et al. ${ }^{[59]}$ have shown that hippocampal Fos induction, which is associated with a poor performance of aged mice in the water maze test is altered in the absence of molar teeth. In addition, decreased synthesis ${ }^{[6-68]}$ and release of acetylcho- 
line ${ }^{[10,68]}$ and dopamine ${ }^{[44]}$ concomitant with reduced levels of synaptophysin have been observed in the hippocampus following removal of molar teeth [68].

Clinically, drugs that promote cholinergic activity contribute to reduction of cognitive deficits ${ }^{[69]}$. Dopaminergic inputs to the hippocampus originate in the ventral tegmental area and are important for hippocampal function. According to Kushida et al. ${ }^{[44]}$, masticatory deficits were associated with reduced dopamine levels in the hippocampus, but there were no effects on dopaminergic synthesis, suggesting that hippocampal damage was related to the reduction in dopamine release from local dopaminergic terminals but not to reduced dopamine synthesis.

Dopamine reduction impairs cognition in both rodents ${ }^{[44]}$ and primates ${ }^{[70]}$ likely contributing to dementia. Combined depletion of dopamine and acetylcholine induces cognitive deficits in neurodegenerative diseases such as Alzheimer's disease [71]. It follows that cholinergic and dopaminergic depletion induced by masticatory deficits may promote or contribute to neurological and cognitive impairment.

The hippocampus is an important neurogenic region of the adult $\mathrm{CNS}^{[72]}$. It has been reported that reduction of chewing promotes suppression of cell proliferation in the hippocampal dentate gyrus, a source of new neurons in adult rodents ${ }^{[73,74]}$. Some experimental evidence suggests that hippocampal neurogenesis plays a role in learning and memory, even in humans ${ }^{[75,76]}$. Some authors have suggested that neurogenesis is regulated at the systemic level, and that physical activity leads to increased neurogenic potential. It is possible that activity of masticatory muscles during the act of chewing may be sufficient to influence cell proliferation and neurogene$\operatorname{sis}^{[74]}$.

\section{Occlusal disharmony induces deterioration of hippocampal cells}

Hormones may be involved in the mechanisms underlying the effects of masticatory hypofunction on cognition. Loss of molar teeth was associated with increased adrenal volume in mice ${ }^{[58]}$. It has been hypothesized that increased plasma levels of corticosterone promote hippocampal neuronal damage ${ }^{[77]}$. In this experimental condition, stress in the oral cavity was observed, especially in the periodontal mechanoreceptors, which are more sensitive to corticosterone than any other type of somatosensory receptor ${ }^{[77]}$.

An experimental model was created with the purpose of inducing a form of occlusal disharmony in the stomatognathic system ${ }^{[78]}$. The researchers induced an increase in vertical occlusion by applying acrylic caps to the lower incisors of rats. This occlusal disharmony was intended to model chronic stress in the stomatognathic system. Increased levels of plasma corticosteroids might suppress learning and memory, given that the hippocampus is a major target for corticosteroid actions and is one of the CNS regions that contribute to the regulation of the hypothalamo-pituitary-adrenal axis [78].

Kubo et al. ${ }^{[79]}$ found increased plasma levels of corticosterone after vertical occlusion in aged mice. Similar results were obtained by Ichisachi et al..80]. These authors reported decreased mRNA expression and immunoreactivity for glucocorticoid receptors in both hippocampal CA1 and the dentate gyrus. In fact, the extraction of molar teeth in aged mice increased plasma levels of corticosterone and was associated with deterioration of hippocampal neurons, glial cells and spatial memory ${ }^{[56-59]}$. Furthermore, Onozuka et al.[58] reported that the glucocorticoids - such as cortisol and corticosterone - secreted by the adrenal cortex, inhibited the production of new neurons to suppress the proliferation of granule cells in the dentate gyrus region in adult mice. It is likely that the activity of masticatory muscles is one of the factors regulating cell proliferation in the hippocampal dentate gyrus. It follows that modulation of corticoid levels as a result of masticatory alterations such as those induced by the loss of molars or by changing the occlusion, may lead to loss of neurons and inhibition of hippocampal neurogenesis resulting in cognitive impairment ${ }^{[10,46]}$.

\section{Masticatory stimulation plays a pivotal role on cognitive functions}

From the studies described above it can be inferred that regular sensory stimulation in the course of mastication is essential for maintaining the learning and memory functions of the aged hippocampus ${ }^{[9]}$. The decrease of masticatory stimulation, among other factors, may reduce synaptic density in the cerebral cortex by decreasing sensory input to the cerebral cortex ${ }^{[46]}$. This supports the hypothesis that regulation of sensory input from the masticatory organs is important for maintaining cognitive functions, especially in the aged hippocampus ${ }^{[9]}$.

Cerebellar functions also seem to be influenced by masticatory activity ${ }^{[12]}$. Quintero et al. ${ }^{[81]}$ have reported functional connections between the cerebellum and cortical areas, including sensorimotor and cingulate cortices, during masticatory activity. It has been well documented that the cerebellum is responsible for motor planning, including the force needed to perform motor tasks [82]. In terms of oral functions, studies in humans ${ }^{[81]}$ and animals[83] have demonstrated that the cerebellum may be involved in chewing rhythmicity and motor control motivated by proprioceptive inputs. The increase in cerebellar ac- 
tivity during chewing may improve certain motor functions related to this motor behavior ${ }^{[84]}$.

\section{Conclusion}

In conclusion, mastication is correlated with health in general as well as with digestive processes and peripheral sensory and motor input to the brain likely rendering physiological benefits to CNS cognitive areas. During chewing PFC, sensory and insular cortices, striatum, thalamus, cerebellum and hippocampus show increases in basal blood flow, which is related to increased supply of oxygen and glucose, both essential for brain function.

Taken together, the data suggest that mastication plays a role in cognitive functions and its impairment may constitute a risk factor for dementia and chronic neurodegenerative diseases associated with aging.

Further studies are necessary to elucidate the neural basis of the involvement of other brain structures in masticatory function. The involvement of motor areas (motor cortex and cerebellum) and memory-related areas (striatum and PFC) should be a target for future research. Investigations of anatomical and functional correlations between masticatory function and the hippocampus, extending existing observations of morphological and functional impairments related to masticatory deficits would also be of interest and might contribute to the development of therapies to prevent or minimize cognitive dysfunction caused by masticatory hypofunction.

\section{Competing Interests}

The authors have declared that no competing interest exists.

\section{References}

1. United Nations. World population ageing 1950-2050. New York: United Nations 2007.

2. Instituto Brasileiro de Geografia e Estatística. Projeção da população do Brasil por sexo e idade para o período 1980-2050: revisão 2004. ftp://ftp.ibge.gov.br/Estimativas_Projecoes_Populacao/Revisao2004_Projec oes_1980 2050.

3. Sequeira E, Neves DM, Brunetti RF, Luz DT, Brunetti FL. Odontogeriatria: a especialidade do futuro. Rev ABO Nac. 2001; 9: 72-78.

4. United Nations Population Division. World population prospects: the 2002 revision. New York: United Nations 2003.

5. Nakata M. Masticatory function and its effects on general health. Int Dent J. 1998; 48: 540-548.

6. Miura H, Miura K, Mizugai H, Arai Y, Umenai T, Isogai E. Chewing ability and quality of life among the elderly residing in a rural community in Japan. J Oral Rehabil. 2000; 27: 731-734

7. Miura H, Yamasaki K, Kariyasu M, Miura K, Sumi Y. Relationship between cognitive function and mastication in elderly females. J Oral Rehabil. 2003; 30: 808-811.

8. Scherder E, Posthuma W, Bakker T, Vuijk PJ, Lobbezoo F. Functional status of masticatory system, executive function and episodic memory in older persons. J Oral Rehabil. 2008; 35: 324-336.

9. Ono Y, Yamamoto T, Kubo K, Onozuka M. Occlusion and brain function: mastication as a prevention of cognitive dysfunction. J Oral Rehabil. 2010; 1: $1-17$.

10. Kato T, Usami T, Noda Y, Hasegawa M, Ueda M, Nabeshima T. The effect of the loss of molar teeth on spatial memory and acetylcholine release from the parietal cortex in aged rats. Behav Brain Res. 1997; 83: 239-242

11. Momose I, Nishikawa J, Watanabe T, et al. Effect of mastication on regional cerebral blood flow in humans examined by positron-emission tomography with 15O-labelled water and magnetic resonance imaging. Arch Oral Biol. 1997; 42: 57-61.

12. Onozuka M, Fujita M, Watanabe $K$, et al. Mapping brain region activity during chewing: a functional magnetic resonance imaging study. J Dent Res. 2002; 81: 743-746.

13. Onozuka M, Fujita M, Watanabe K, et al. Age-related changes in brain regional activity during chewing: a functional magnetic resonance imaging study. J Dent Res. 2003; 82: 657-660.

14. Nakamura Y, Katakura N. Generation of masticatory rhythm in the brainstem. Neurosci Res. 1995; 23: 1-19.

15. Onozuka M, Hirano $\mathrm{Y}$, Tachibana A, et al. Interactions between chewing and brain activities in humans. In: Onozuka M, Yen CT, eds. Novel trends in brain science. Tokyo: Springer 2007; 99-113.

16. Hirano $\mathrm{Y}$, Obata $\mathrm{T}$, Kashikura $\mathrm{K}$, et al. Effects of chewing in working memory processing. Neurosci Lett. 2008; 436: 189-192.

17. Milner B, Squire LR, Kandel ER. Cognitive neuroscience and the study of memory. Neuron. 1998; 20: 445-468.

18. Packard MG, McGaugh JL. Double dissociation of fornix and caudate nucleus lesions on acquisition of two water maze tasks: further evidence for multiple memory systems. Behav Neurosci. 1992; 106: 439-446.

19. McDonald RJ, White NM. A triple dissociation of memory systems: hippocampus, amygdala, and dorsal striatum. Behav Neurosci. 1993; 107: 3-22.

20. Yin HH, Knowlton BJ. The role of the basal ganglia in habit formation. Nat Rev Neurosci. 2006; 7: 464-476.

21. Middleton FA, Strick PL. Basal-ganglia 'projections' to the prefrontal cortex of the primate. Cereb Cortex. 2002; 12: 926-935

22. Packard MG, Knowlton BJ. Learning and memory functions of the basal ganglia. Annu Rev Neurosci. 2002; 25: 563-593.

23. Graybiel, AM. The basal ganglia and chunking of action repertoires. Neurobiol Learn Mem. 1998; 70: 119-136.

24. Bar-Gad I, Morris G, Bergman H. Information processing, dimensionality reduction and reinforcement learning in the basal ganglia. Prog Neurobiol. 2003; 71: 439-473.

25. Pasupathy A, Miller EK. Different time courses oflearning-related activity in the prefrontal cortex and striatum. Nature. 2005; 433: 873-875.

26. Moser E, Moser MB, Andersen P. Spatial learning impairment parallels the magnitude of dorsal hippocampal lesions, but is hardly present following ventral lesions. J Neurosci. 1993; 13: 3916-3925.

27. Bannerman DM, Rawlins JN, McHugh SB, et al. Regional dissociations within the hippocampus: memory and anxiety. Neurosci Biobehav Rev. 2004; 28: 273-283.

28. Alexander GE, Crutcher MD, DeLong MR. Basal ganglia-thalamocortical circuits: parallel substrates for motor, oculomotor, 'prefrontal' and 'limbic' functions. Prog Brain Res. 1990; 85: 119-146.

29. Nambu, A. Seven problems on the basal ganglia. Curr Opin Neurobiol. 2008;18: 595-604

30. Kopell BH, Greenberg BD. Anatomy and physiology of the basal ganglia: implications for DBS in psychiatry. Neurosci Biobehav Rev. 2008; 32: 408-422.

31. Grassi G, Setavalle G, Dell'Oro R, Mancia G. Sympathetic mechanisms, organ damage, and antihypertensive treatment. Curr Hypertens Rep. 2011; 13: 303-308.

32. Stephens R, Tunney RJ. Role of glucose in chewing gum related facilitation of cognitive function. Appetite. 2004; 43: 211-213.

33. Wilkinson L, Scholey A, Wesnes K. Chewing gum selectively improves aspects of memory in healthy volunteers. Appetite. 2002; 38: 235-236.

34. Ono Y, Dowaki K, Ishiyama A, Onozuka M. Gum chewing maintains working memory acquisition. Int J Bioelectromagnetism. 2009; 11: 130-134.

35. Squire LR. Memory and the hippocampus: a synthesis of findings with rats, monkeys and humans. Psychol Rev. 1992; 99: 195-221.

36. Cherkin A. Kinetics of memory consolidation: role of amnesic treatment parameters. Proc Natl Acad Sci USA.1968; 63: 1094-1101.

37. Izquierdo I, Medina JH. Memory formation: the sequence of biochemical events in the hippocampus and its connection to activity in other brain structures. Neurobiol Learn Mem. 1997; 68: 285-316.

38. McGaugh JL. Time-dependent processes in memory storage. Science. 1966; 153: 1351-1359.

39. McGaugh JL. A multi-trace view of memory storage processes. Accademia Nazionale dei Lincei. 1968; 109: 13-28.

40. Gold PE, McGaugh JL. A single-trace, two process view of memory storage processes. In Short-Term Memory. Edited by Deutsch D, Deutsch JA. 1975; 140: 197-216.

41. Hirano Y, Obata T, Takahashi H, Tachibana A, Kuroiwa D, Takahashi T, Ikehira $\mathrm{H}$, Onozuka M. Effects of chewing on cognitive processing speed. Brain Cogn. 2013; 8: 376-81.

42. Baker JR, Bezance JB, Zellaby E, Aggleton JP. Chewing gum can produce context-dependent effects upon memory. Appetite. 2004; 43: 207-210.

43. Onyper SV, Carr TL, Farrar JS, Floyd BR. Cognitive advantages of chewing gum. Now you see them, now you don't. Appetite. 2011; 57: 321-8.

44. Kushida S, Kimoto K, Hori N, et al. Soft-diet feeding decreases dopamine release and impairs aversion learning in Alzheimer model rats. Neurosci Lett. 2008; 439: 208-211.

45. Ekuni D, Tomofuji T, Irie K, et al. Occlusal disharmony increases amyloid- $\beta$ in the rat hippocampus. Neuromolecular Med. 2011; 13: 197-203. 
46. Yamamoto T, Hirayama A. Effects of soft-diet feeding on synaptic density in the hippocampus and parietal cortex of senescence-accelerated mice. Brain Res. 2001; 902: 255-263.

47. Stein PS, Desrosiers M, Donegan SJ, Yepes JF, Kryscio RJ. Tooth loss, dementia and neuropathology in the Nun study. J Am Dent Assoc. 2007; 138: 1314-1322.

48. Yamamoto T, Kondo K, Hirai H, Nakade M, Aida J, Hirata Y. Association between self-reported dental health status and onset of dementia: a 4-year prospective cohort study of older Japanese adults from the Aichi Gerontological Evaluation Study (AGES) Project. Psychosom Med. 2012; 74: 241-8.

49. Lexomboon D, Trulsson M, Wårdh I, Parker MG. Chewing ability and tooth loss: association with cognitive impairment in an elderly population study. J Am Geriatr Soc. 2012; 60: 1951-1956.

50. Shimazaki Y, Soh I, Yamashita T, Koga T, Miyazaki H, Takehara T. Influence of dentition status on physical disability, mental impairment, and mortality in institutionalized elderly. J Dent Res. 2001; 80: 340-345.

51. Miura H, Araki Y, Hirai T, Isogai E, Hirose K, Umanai T. Evaluation of chewing activity in the elderly person. J Oral Rehabil. 1998; 25: 190.

52. Kondo K, Niino M, Shido K. A case-control study of Alzheimer's disease in Japan-significance of life-styles. Dementia. 1994; 5: 314-326.

53. Gatz M, Mortimer JA, Fratiglioni L, et al. Potentially modifiable risk factors for dementia in identical twins. Alzheimer's \& Dementia. 2006; 2: 110-117.

54. Muramoto T, Takano Y, Soma K. Time-related changes in periodontal mechanoreceptors in rat molars after the loss of occlusal stimuli. Arch Histol Cytol. 2000; 63: 369-380.

55. Onozuka M, Watanabe K, Mirbod SM, et al. Reduced mastication stimulates impairment of spatial memory and degeneration of hippocampal neurons in aged SAMP8 mice. Brain Res. 1999; 826: 148-153.

56. Onozuka M, Watanabe K, Nagasaki S, et al. Impairment of spatial memory and changes in astroglial responsiveness following loss of molar teeth in aged SAMP8 mice. Behav Brain Res. 2000; 108: 145-155.

57. Watanabe K, Tonosaki K, Kawase T, et al. Evidence for involvement of dysfunctional teeth in the senile process in the hippocampus of SAMP8 mice. ExpGerontol. 2001; 36: 283-295.

58. Onozuka M, Watanabe $\mathrm{K}$, Fujita $\mathrm{M}$, Tonosaki $\mathrm{K}$, Saito S. Evidence for involvement of glucocorticoid response in the hippocampal changes in aged molarless SAMP8 mice. Behav Brain Res. 2002; 131: 125-129.

59. Watanabe K, Ozono S, Nishiyama K, et al. The molarless condition in aged SAMP8 mice attenuates hippocampal Fos induction linked to water maze performance. Behav Brain Res. 2002; 128: 19-25.

60. Kubo KY, Iwaku F, Watanabe K, Fujita M, Onozuka M. Molarless-induced changes of spines in hippocampal region of SAMP8 mice. Brain Res. 2005; 1057: 191-195.

61. Squire LR, Stark CE, Clark RE. The medial temporal lobe. Annual Review Neuroscience. 2004; 27: 279-306

62. Adams MM, Shi L, Linville MC, et al. Caloric restriction and age affect synaptic proteins in hippocampal CA3 and spatial learning ability. ExpNeurol. 2008; 211: 141-149.

63. Schipper HM, Bennett DA, Liberman A, et al. Glial heme oxygenase-1 expression in Alzheimer disease and mild cognitive impairment. Neurobiol Aging. 2006; 27: 252-261.

64. Moore AH, Wu M, Shaftel SS, Graham KA, O'Banion MK. Sustained expression of interleukin-1beta in mouse hippocampus impairs spatial memory. Neuroscience. 2009; 164: 1484-1495

65. Slezak M, Pfrieger FW, Soltys Z. Synaptic plasticity, astrocytes and morphological homeostasis. J Physiol Paris. 2006; 99: 84-91.

66. Makiura T, Ikeda Y, Hirai T, Terasawa H, Hamaue N, Minami M. Influence of diet and occlusal support on learning memory in rats behavioral and biochemical studies. Res Commun Mol Pathol Pharmacol. 2000; 107: 269-277.

67. Terasawa $\mathrm{H}$, Hirai $\mathrm{T}$, Ninomiya $\mathrm{T}$, et al. Influence of tooth-loss and concomitant masticatory alterations on cholinergic neurons in rats: immunohistochemical and biochemical studies. Neurosci Res. 2002; 43:373-379.

68. Onozuka M, Watanabe K, Fujita M, Tomida M, Ozono S. Changes in the septohippocampal cholinergic system following removal of molar teeth in the aged SAMP8 mouse. Behav Brain Res. 2002; 133: 197-204.

69. Winblad B, Engedal $\mathrm{K}$, Soininen $\mathrm{H}$, et al. A 1-year, randomized, placebocontrolled study of donepezil in patients with mild to moderate AD. Neurology. 2001; 57: 489-495.

70. Decamp E, Tinker JP, Schneider JS. Attentional cueing reverses deficits in spatial working memory task performance in chronic low dose MPTP-treated monkeys. Behav Brain Res. 2004;152: 259-62.

71. Bosboom JL, Stoffers D, Wolters EC. The role of acetylcholine and dopamine in dementia and psychosis in Parkinson's disease. J Neural Transm Suppl. 2003; 65: 185-95.

72. Spalding KL, Bergmann O, Alkass K, et al. Dynamics of hippocampal neurogenesis in adult humans. Cell 2013; 153: 1219-1227.

73. Aoki H, Kimoto K, Hori N, Toyoda M. Cell proliferation in the dentate gyrus of rat hippocampus is inhibited by soft diet feeding. Gerontology. 2005; 51 : 369-374.

74. Mitome $\mathrm{M}$, Hasegawa $\mathrm{T}$, Shirakawa $\mathrm{T}$. Mastication influences the survival of newly generated cells in mouse dentate gyrus. Neuroreport. 2005; 16: 249-252.

75. Zhao C, Deng W, Gage FH. Mechanisms and functional implications of adult neurogenesis. Cell. 2008; 132: 645-660.

76. Abrous DN, Koehl M, Le Moal M. Adult neurogenesis: from precursors to network and physiology. Physiol Rev. 2005; 85: 523-569.
77. Lanfumey L, Mongeau R, Cohen-Salmon C, Hamon M. Corticosteroid-serotonin interactions in the neurobiological mechanisms of stress-related disorders. Neurosci Bio behav Rev. 2008; 32: 1174-1184.

78. Yoshihara T, Matsumoto Y, Ogura T. Occlusal disharmony affects plasma corticosterone and hypothalamic noradrenaline release in rats. J Dent Res. 2001; 80: 2089-2092.

79. Kubo KY, Yamada Y, Iinuma M, et al. Occlusal disharmony induces spatial memory impairment and hippocampal neuron degeneration via stress in SAMP8 mice. Neurosci Lett. 2007; 414: 188-191.

80. Ichihashi $Y$, Arakawa $Y$, Iinuma M, et al. Occlusal disharmony attenuates glucocorticoid negative feedback in aged SAMP8 mice. Neurosci Lett. 2007; 427: 71-76.

81. Quintero A, Ichesco E, Schutt R, Myers C, Peltier S, Gerstner GE. Functional connectivity of human chewing: An fcMRI study. J Dent Res. 2013; 92: 272-278.

82. Manto M, Bower JM, Conforto AB, et al. Consensus paper: roles of the cerebellum in motor control--the diversity of ideas on cerebellar involvement in movement. Cerebellum. 2012; 11: 457-87.

83. Byrd KE, Luschei ES. Cerebellar ablation and mastication in the guinea pig (Cavia porcellus). Brain Res. 1980; 197: 577-81.

84. Takahashi T, Miyamoto T, Terao A, Yokoyama A. Cerebral activation related to the control of mastication during changes in food hardness. Neuroscience 2007; 145: 791-794. 